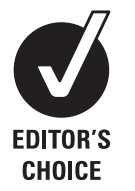

See Editorial Commentary,
p 239

${ }^{1}$ University Department of Clinical Neurosciences, Stroke Prevention Research Unit, John Radcliffe Hospital, Oxford, UK ${ }^{2}$ Department of Neurology, Stoke Mandeville Hospital, Aylesbury, UK

\section{Correspondence to} Professor Peter M Rothwell, Department of Clinical Neurology, Stroke Prevention Research Unit, John Radcliffe Hospital, Level 6, West Wing, Headley Way, Oxford OX3 9DU, UK; peter.rothwell@clneuro.ox.ac.uk

Received 2 December 2008 Revised 31 March 2009 Accepted 8 June 2009

\title{
The SCAN rule: a clinical rule to reduce CT misdiagnosis of intracerebral haemorrhage in minor stroke
}

\author{
C E Lovelock, ${ }^{1}$ J N Redgrave, ${ }^{1}$ D Briley, ${ }^{2}$ P M Rothwell ${ }^{1}$
}

\section{ABSTRACT}

Many patients with minor stroke are referred to outpatient clinics and are not scanned immediately. A clinical rule is needed to identify patients who are likely to have intracerebral haemorrhage (ICH) and require urgent brain imaging and patients who can safely start antiplatelet agents before scanning.

Methods Clinical factors associated with ICH were determined in 334 consecutive patients with minor stroke (National Institute of Health Stroke Scale score $\leq 3$ ), and a predictive model for ICH that was validated in a cohort of 280 patients presenting to a hospital-stroke clinic was derived. Prognostic value was quantified as the area under the ROC curve (c statistics).

Results The proportion of ICH in minor stroke was $5.1 \%$ $(95 \% \mathrm{Cl} 3.2 \%$ to $8.0 \%)$ in OXVASC, and $5.4 \%$ (3.3\% to $8.7 \%)$ in the clinic cohort. Clinical factors predictive of ICH in OXVASC included blood pressure on initial assessment $\geq 180 / 110 \mathrm{~mm} \mathrm{Hg}$ (OR 14.5, 95\% Cl 1.8 to 114 , $\mathrm{p}=0.001$ ), vomiting (OR 15.7, 95\% Cl 5.4 to $46, p<0.001$ ), confusion (OR 8.2, 95\% Cl 2.9 to $23, \mathrm{p}<0.001$ ) and anticoagulation use (OR 7.8, 95\% $\mathrm{Cl} 2.2$ to $28, \mathrm{p}=0.006$ ), and at least one predictive factor was identified in all 17 patients with ICH and in 35\% overall (c statistic 0.92, 95\% $\mathrm{Cl} 0.88$ to 0.97). Therefore, we derived the SCAN rule to identify ICH if $\geq 1$ of the following were present: (S) severe hypertension, (C) confusion, (A) anticoagulation, (N) nausea and vomiting. In the clinic validation cohort, $\geq 1$ predictive factor was identified in 14/15 of patients with $\mathrm{ICH}$ and in $24 \%$ overall (c statistic $0.87,95 \% \mathrm{Cl} 0.79$ to 0.95 ).

Conclusion The SCAN rule appears to be specific and sensitive at identifying $\mathrm{ICH}$ in an independent cohort of patients with minor stroke, although further independent validations are needed.

In the UK and elsewhere, ${ }^{1} 2$ many patients with minor stroke are not admitted to hospital but are assessed and investigated in specialist clinics. Although current guidelines recommend that highrisk patients should be assessed by a specialist within $24 \mathrm{~h}$ of symptom onset and everyone should be seen within 7 days, ${ }^{3}$ currently, only $50 \%$ of referrals to neurovascular clinics in the UK are seen within 7 days, ${ }^{4}$ with many patients waiting substantially longer for brain imaging.

Delays to assessment and brain imaging, in particular, can have a number of consequences. First, referring physicians may be reluctant to start antiplatelet therapy until intracerebral haemorrhage (ICH) has been excluded radiologically, even though the prompt initiation of secondary stroke prevention including antiplatelet agents is known to reduce early ischaemic stroke recurrence. ${ }^{5} 6$
Second, if late presenting patients are only scanned with CT, which is insensitive to acute haemorrhage after $7-10$ days, ${ }^{7-9}$ then cases with primary ICH may be missed. An earlier clinic-based study (of patients presenting at $\geq 4$ days post-stroke) using concurrent MRI and CT imaging, showed that 75\% with primary ICH identified on MRI were misdiagnosed as infarction on CT because of delays to scanning. ${ }^{7}$ Consequently, these patients might have been started on inappropriate and potentially harmful long-term prevention therapy with antithrombotic agents, and MRI was recommended for all late-presenting patients. However, this policy may be difficult to achieve when access to MRI is limited, and a system for identifying which patients with minor stroke are most likely to have had a recent ICH on clinical grounds would be useful.

Previous scoring systems have been created to identify patients with $\mathrm{ICH}^{10}{ }^{11}$ but these are weighted heavily on signs of major ICH including coma. These scores have been criticised for not being adequately sensitive in detecting $\mathrm{ICH}^{12}$ and are unlikely to be useful in patients presenting with minor stroke. We aimed to identify clinical factors that might predict the presence or absence of $\mathrm{ICH}$ in patients with minor stroke and could be used to determine patients who could be safely treated with antiplatelet agents before assessment in clinic and those who needed urgent brain imaging before treatment.

\section{METHODS}

Patients with minor stroke were prospectively ascertained from separate derivation and validation cohorts, defining minor stroke as any stroke meeting the World Health Organization criteria ${ }^{13}$ with a National Institute of Health Stroke Scale score ${ }^{14}$ on assessment of $\leq 3$. The derivation cohort included consecutive patients prospectively ascertained from the first 5 years of the Oxford Vascular Study (OXVASC). OXVASC is a population-based study of all incident or recurrent acute vascular events including transient ischaemic attack (TIA) and stroke, in an Oxfordshire population of around 91000 individuals registered with 63 family physicians, the methods of which have been described in detail elsewhere. ${ }^{15}$

The validation cohort comprised consecutive patients with minor stroke, who had been referred by their general practitioners from 2000 to 2006 to a "TIA" clinic in a district general hospital located in a neighbouring county. Nearly all of the patients in this cohort were scanned with MRI on the same day as the clinic assessment. The MRI scanner was a 1.5-T Siemens Symphony system using an axial turbo gradient spin echo sequence (TGSE) 
(repetition time (TR) $4000 \mathrm{~ms}$, echo time (TE) $95 \mathrm{~ms}, 19$ slices, slice thickness $6.0 \mathrm{~mm}$, matrix $256 \times 256$, FOV $230 \times 230$ ).

In both cohorts, details of each patient's presenting symptoms and examination findings as well as background vascular risk factors were recorded by a study neurologist. Stroke subtyping was based on the results of the first scan taken post-stroke, and classified as being consistent with ischaemic stroke, ICH, or haemorrhagic transformation of infarction. For the purpose of later comparisons between ischaemic stroke and $\mathrm{ICH}$, cases of haemorrhagic transformation of infarction were included in the group with ischaemic stroke, as most cases of haemorrhagic transformation of infarction only involve petechial haemorrhage into the region of infarction, and this relatively frequent "complication" is often asymptomatic ${ }^{16}$ and does not usually influence the decision to use antithrombotic therapy in the longer term. Cases of haemorrhage secondary to trauma, intracranial tumour and haematopoietic malignancy, as well as cases of subdural and subarachnoid haemorrhage, were excluded.

Data from the OXVASC cohort were used to derive a model to predict the presence of ICH on imaging. Because we expected a small number of patients with ICH in this cohort, we tried to minimise the possibility of chance associations by testing variables that had been identified as predictors of ICH in previous clinical cores, and which appeared to be relevant to a population with minor stroke. These included variables that had featured in both the Allen score ${ }^{10}$ and Siriraj score, ${ }^{11}$ such as headache or vomiting on presentation and blood pressure at assessment. We also looked for an association between ICH and a history of an acute confusional state with stroke onset, defined in accordance with DSM IV-R criteria ${ }^{17}$ as a history of acute onset reduced attention and disorganised thinking, disorientation or memory disturbance. This had not been included in the above scores, but acute confusional states have been shown to be associated with ICH by previous investigators. ${ }^{18} 19$ Blood pressure values were transformed into categorical variables for ease of use in the model, with cut-offs corresponding to the classification system of the joint European Societies of Cardiology and Hypertension ${ }^{20}$ for increasing levels of hypertension. When variables were missing, these were assumed to be absent and the frequency of missing values never exceeded $5 \%$ of the cohort.

Univariate associations between the presence of ICH and clinical variables were evaluated using Student $t$ test for continuous variables and Fisher's exact test for categorical variables. In addition, the usefulness of each clinical variable as a tool for differentiating between ICH and ischaemic stroke was compared using diagnostic odd ratios (ORs). There was insufficient statistical power to do multivariate modelling on the derivation cohort alone. Instead we continued to add variables associated with $\mathrm{ICH}$ with a significance of $\mathrm{p}<0.1$ to the model in decreasing order of their diagnostic OR, until addition of further variables did not appear to enhance the predictive value of the model.

This model was then internally validated using the OXVASC cohort and externally validated using the hospital clinic cohort. The frequency of ICH in each cohort was stratified according to the number of predictor variables present. Receiver operating characteristic (ROC) curves were then derived by determining the sensitivity and specificity of the model for identifying ICH with increasing numbers of predictor variables. In addition, we pooled data from both cohorts of patients of minor stroke and analysed the independent predictive effect of the variables used in the model.

Finally, we wished to compare the performance of our model in both cohorts against an established and well-known model-the Siriraj score. ${ }^{11}$ This score uses a cut-off of $<-1$ to predict infarction and $>1$ to predict $\mathrm{ICH}$, so that brain imaging to detect $\mathrm{ICH}$ is needed if the score is $\geq 1$. As one of the clinical variables used in the Siriraj score is the patient's level of consciousness at assessment, and many patients in these cohorts presented late to outpatient clinics having already recovered, this variable was redefined as a history of drowsiness at the time of the stroke. The Allen score ${ }^{10}$ was not derived for either cohort as the score depends on examination findings including plantar responses at $24 \mathrm{~h}$ post-stroke, and such data were not complete in the hospital clinic-based cohort.

\section{RESULTS}

In the OXVASC derivation cohort, we identified 412 consecutive patients with minor stroke. Of these, 78 patients were scanned by CT beyond 10 days or declined brain imaging and were excluded from further analysis as ICH could not be reliably diagnosed. $^{7-9}$ Of the remaining 334 patients, who were either scanned with CT within 10 days (median interval (interquartile range, or IOR) of $3(1-5)$ days) or MRI, there were 17 patients with ICH (5.1\%, IOR $3.2 \%$ to $8.0 \%)$ and 4 with haemorrhagic transformation of infarction (1.2\%, IOR $0.5 \%$ to $3.0 \%)$.

In the hospital clinic validation cohort, we identified 284 consecutive patients with minor stroke. Of these, four patients had undergone CT at $>10$ days post-event because of contraindications to MRI and were excluded from further analysis. Of the remaining 280 patients who were scanned with MRI on the day of clinic after a median delay of 15 days (IOR 10-23) following stroke onset, there were 15 patients with ICH $(5.4 \%$, 95\% CI $3.3 \%$ to $8.7 \%$ ) and 6 with haemorrhagic transformation of infarction $(2.1 \%, 95 \%$ CI $1.0 \%$ to $4.6 \%)$.

Baseline characteristics including risk factors, premorbid medication use, and stroke symptoms are shown for both cohorts in table 1 . The OXVASC cohort was slightly older than the hospital clinic cohort but had a similar ratio of male to female patients. The OXVASC cohort had a higher proportion of patients with a past history of TIA $(p=0.002)$, but there were no other significant differences between the cohorts with respect to previously diagnosed comorbidities. Proportions on premorbid anticoagulation therapy were also similar, but more patients in OXVASC were taking antiplatelet agents $(p=0.03)$. Headache $(p=0.02)$ and vomiting $(p=0.006)$ were more commonly identified in the OXVASC cohort, although these symptoms were no less frequent in outpatients compared with inpatients within this cohort. A similar proportion of minor strokes in both cohorts were associated with transient confusion at onset. The mean systolic blood pressure at presentation was higher in the OXVASC cohort $(p<0.001)$, although the rates of previously diagnosed hypertension were similar in both cohorts.

Table 2 shows the diagnostic ORs for ICH versus ischaemic stroke for each of the variables tested in the OXVASC derivation cohort. Three clinical symptoms at stroke onset were predictive for ICH-vomiting (OR 15.7, 95\% CI 5.4 to 46, $\mathrm{p}<0.001$ ), confusion (OR 8.2, 95\% CI 2.9 to $23, \mathrm{p}<0.001)$ and headache (OR $4.9,95 \%$ CI 1.8 to $13, p=0.002)$. Severe hypertension at initial assessment (systolic blood pressure $\geq 180 \mathrm{~mm} \mathrm{Hg}$ or diastolic blood pressure $\geq 110 \mathrm{~mm} \mathrm{Hg}$ ) was most predictive of $\mathrm{ICH}$ (OR 14.5, 95\% CI 1.8 to $114, \mathrm{p}=0.001$ ), and lower cut-off values for blood pressure were less predictive. Premorbid anticoagulant use was the only pre-existing clinical risk factor associated with an increased likelihood of ICH (OR 7.8, 2.2 to 28, p=0.006). Age was not a discriminatory variable, with no difference in the mean age (SD) of patients with ICH or ischaemic stroke (74 (12) vs 74 (11) years, $p=0.88$ ). The diagnostic ORs were derived for the same variables in the validation cohort (also shown in table 2). With the exception of a history of vomiting and headache, all variables that were significantly associated with $\mathrm{ICH}$ in the 
Table 1 Characteristics of patients with minor stroke in the OXVASC derivation cohort and hospital clinic validation cohorts

\begin{tabular}{lccc}
\hline & $\begin{array}{c}\text { OXVASC } \\
\text { (n=334) }\end{array}$ & $\begin{array}{l}\text { Hospital clinic } \\
\text { cohort (n=280) }\end{array}$ & p Value \\
\hline Mean age (SD) & $74(12)$ & $72(10)$ & 0.04 \\
Men (\%) & $195(58)$ & $166(59)$ & 0.87 \\
Mean systolic blood pressure (SD) & $158(30)$ & $150(23)$ & $<0.001$ \\
Mean diastolic blood pressure (SD) & $85(15)$ & $83(12)$ & 0.17 \\
Premorbid risk factors (\%) & & & 0.80 \\
Hypertension & $209(63)$ & $172(61)$ & 0.05 \\
Myocardial infarction & $39(12)$ & $49(18)$ & 0.08 \\
Diabetes & $33(10)$ & $41(15)$ & 0.52 \\
Current smoker & $54(16)$ & $51(18)$ & 0.19 \\
Previous stroke & $50(15)$ & $31(11)$ & 0.002 \\
Previous TIA & $58(17)$ & $24(9)$ & 0.23 \\
Hyperlipidemia & $91(27)$ & $64(23)$ & \\
Premorbid medications (\%) & & & 0.03 \\
Antiplatelet & $148(44)$ & $99(35)$ & 0.85 \\
Anticoagulation & $16(5)$ & $12(4)$ & 0.31 \\
Symptoms at onset & & & 0.006 \\
Confusion & $32(10)$ & $20(7)$ & 0.02 \\
Vomiting & $25^{*}(8)$ & $7(3)$ & \\
Headache & $68^{* *}(20)$ & $37(13)$ & \\
\hline *Twelve of these were clinic pationts & & & \\
$*$ & & & \\
\hline
\end{tabular}

*Twelve of these were clinic patients.

**Thirty-five of these were clinic patients.

derivation cohort were also significantly associated with ICH in the validation cohort.

A history of headache at stroke onset had the lowest diagnostic OR of all the predictor variables that were significant on univariate testing in the derivation cohort, and adding this variable to our model did not improve the sensitivity but reduced the specificity. Therefore. we only selected the four variables that appeared to be most useful in the prediction of $\mathrm{ICH}$-severe hypertension at initial assessment, premorbid anticoagulation, a history at presentation of transient confusion and vomiting at stroke onset. Table 3 shows the percentage risk of having ICH stratified according to the number of predictor variables in the OXVASC and hospital clinic cohort. In OXVASC, at least one clinical predictor was present in 116 (35\%) patients with any minor stroke but in all patients with ICH. If two or more clinical predictors were present, $42 \%$ of patients had evidence of a recent haemorrhage on scan.

In the hospital clinic validation cohort, at least one clinical predictor was present in $66(24 \%)$ patients but in $14(93 \%)$ patients with ICH. If two or more clinical predictors were present, $25 \%$ of patients had evidence of $\mathrm{ICH}$ on scan. ROC areas were 0.92 (95\% CI 0.88 to 0.97 ) and 0.87 (95\% CI 0.79 to $0.95)$ for the derivation and validation cohorts, respectively. In the pooled data set comprising 31 cases of ICH, ICH was only identified in $0.2 \%$ of all patients in whom all clinical predictors were absent. In a multivariate analysis of the pooled data set that included the four predictor variables identified in the derivation cohort, all remained independently predictive of ICH.

Based on these findings, we derived the SCAN rule (table 4), in which scanning with expeditious CT, or MRI for late presenting patients, was recommended when one or more of the following variables were present: severe hypertension at assessment (systolic blood pressure $\geq 180 \mathrm{~mm} \mathrm{Hg}$ or diastolic blood pressure $\geq 110 \mathrm{~mm} \mathrm{Hg}$ ), confusion at onset, anticoagulation, nausea and vomiting at onset. We then compared this rule to the Siriraj score, which predicts absence of ICH in patients with a score of $<-1$. In the OXVASC cohort, use of the Siriraj score would have missed 5 patients (29\%) with ICH. However, in the hospital TIA clinic cohort, only scanning patients with a Siriraj score of $\geq-1$, which is the recommended cut-off for identifying any potential cases with $\mathrm{ICH}$, would have missed 13 patients (87\%) with $\mathrm{ICH}$.

\section{DISCUSSION}

We have derived and validated a rule in which the identification of at least one of four predictor variables, severe hypertension at

Table 2 Diagnostic ORs and $95 \% \mathrm{Cl}$ for predicting ICH versus ischaemic stroke in relation to potential clinical predictors in the OXVASC derivation cohort and the hospital clinic-based validation cohort

\begin{tabular}{|c|c|c|c|c|c|c|c|c|}
\hline & \multicolumn{4}{|c|}{ Derivation cohort } & \multicolumn{4}{|c|}{ Validation cohort } \\
\hline & $\begin{array}{l}\text { Infarct (n/\%) } \\
\mathrm{n}=\mathbf{3 1 7}\end{array}$ & $\begin{array}{l}\text { ICH }(n / \%) \\
n=17\end{array}$ & $\begin{array}{l}\text { Diagnostic OR } \\
\text { (95\% CI) }\end{array}$ & p Value & $\begin{array}{l}\text { Infarct (n/\%) } \\
\mathrm{n}=265\end{array}$ & $\begin{array}{l}\text { ICH }(n / \%) \\
n=15\end{array}$ & $\begin{array}{l}\text { Diagnostic OR } \\
\text { (95\% CI) }\end{array}$ & p Value \\
\hline \multicolumn{9}{|l|}{ Demographic data } \\
\hline Male & $184(58)$ & $11(65)$ & $1.3(0.5$ to 3.7$)$ & 0.63 & $158(60)$ & $8(53)$ & $0.6(0.2$ to 1.7$)$ & 0.79 \\
\hline Mean age (SD) & 74 (12) & 74 (11) & - & 0.88 & $72(11)$ & $73(5)$ & - & 0.59 \\
\hline \multicolumn{9}{|l|}{ Symptoms } \\
\hline Confusion & $25(8)$ & $7(41)$ & $8.2(2.9$ to 23$)$ & $<0.001$ & $14(5)$ & $6(40)$ & 12.0 (3.7 to 38$)$ & $<0.001$ \\
\hline Headache & 59 (19) & $9(53)$ & 4.9 (1.8 to 13$)$ & 0.002 & 34 (13) & $3(20)$ & $1.7(0.5$ to 6.3$)$ & 0.43 \\
\hline \multicolumn{9}{|l|}{ Vascular risk factors } \\
\hline Hyperlipidemia & $86(27)$ & $5(29)$ & $1.1(0.4$ to 3.3$)$ & 0.79 & $60(23)$ & $4(27)$ & $1.2(0.4$ to 4.0$)$ & 0.75 \\
\hline Previous MI & $38(12)$ & $1(6)$ & 0.5 (0.1 to 3.6$)$ & 0.70 & 47 (18) & $2(13)$ & 0.7 (0.2 to 3.3$)$ & 1.00 \\
\hline Previous stroke & 46 (15) & $4(24)$ & 1.8 (0.6 to 5.8 ) & 0.30 & $28(11)$ & $3(20)$ & 2.1 (0.6 to 8.0$)$ & 0.22 \\
\hline Previous TIA & $56(18)$ & $2(12)$ & $0.6(0.1$ to 2.8$)$ & 0.75 & $23(9)$ & $1(7)$ & $0.8(0.1$ to 6.0$)$ & 1.00 \\
\hline Anticoagulation & $12(4)$ & $4(24)$ & $7.8(2.2$ to 28$)$ & 0.006 & $8(3)$ & $4(27)$ & 11.7 (3.0 to 45$)$ & 0.002 \\
\hline \multicolumn{9}{|l|}{ Blood pressure at assessment } \\
\hline$<140 / 90$ & $81(26)$ & $1(6)$ & 1.0 (ref) & & $151(58)$ & $7(47)$ & 1.0 (ref) & \\
\hline $140 / 90-159 / 99 \mathrm{~mm} \mathrm{Hg}$ & $95(30)$ & $2(12)$ & $1.7(0.2$ to 19$)$ & 1.00 & $14(5)$ & $0(0)$ & - & 1.00 \\
\hline $160 / 100-179 / 109 \mathrm{~mm} \mathrm{Hg}$ & $74(23)$ & $2(12)$ & $2.2(0.2$ to 25$)$ & 0.61 & $70(26)$ & $3(20)$ & $0.9(0.2$ to 3.7$)$ & 1.00 \\
\hline$\geq 180 / 110 \mathrm{~mm} \mathrm{Hg}$ & $67(21)$ & $12(70)$ & 14.5 (1.8 to 114$)$ & 0.001 & $30(11)$ & $5(33)$ & 3.6 (1.1 to 12$)$ & 0.04 \\
\hline
\end{tabular}

${ }^{*}$ Excludes individuals on warfarin. 
Table 3 Probability of ICH stratified by number of predictor variables in the OXVASC derivation and the hospital clinic validation cohorts

\begin{tabular}{lccc}
\hline $\begin{array}{l}\text { Number of predictor } \\
\text { variables }\end{array}$ & Patients (\%) & ICH (\%) & $\begin{array}{l}\text { \% Probability of } \\
\text { ICH (95\% Cl) }\end{array}$ \\
\hline OXVASC & & & \\
0 & $218(65 \%)$ & $0(0 \%)$ & $0 \%(0 \%$ to $2 \%)$ \\
1 & $90(27 \%)$ & $6(35 \%)$ & $7 \%(3 \%$ to $14 \%)$ \\
$\geq 1$ & $116(35 \%)$ & $17(100 \%)$ & $15 \%(9 \%$ to $22 \%)$ \\
$\geq 2$ & $26(8 \%)$ & $11(65 \%)$ & $42 \%(26-61 \%)$ \\
Total & $334(100 \%)$ & $17(100 \%)$ & $5 \%(3 \%$ to $8 \%)$ \\
Hospital clinic & & & \\
0 & $214(76 \%)$ & $1(7 \%)$ & $0.5 \%(0 \%$ to $3 \%)$ \\
1 & $58(21 \%)$ & $12(80 \%)$ & $21 \%(12 \%$ to $33 \%)$ \\
$\geq 1$ & $66(24 \%)$ & $14(93 \%)$ & $21 \%(13 \%$ to $33 \%)$ \\
$\geq 2$ & $8(3 \%)$ & $2(13 \%)$ & $25 \%(7 \%$ to $59 \%)$ \\
Total & $280(100 \%)$ & $15(100 \%)$ & $5 \%(3 \%$ to $9 \%)$ \\
Pooled data set & & & \\
0 & $432(70 \%)$ & $1(3 \%)$ & $0.2 \%(0 \%$ to $1 \%)$ \\
1 & $148(24 \%)$ & $18(56 \%)$ & $12 \%(8 \%$ to $18 \%)$ \\
$\geq 1$ & $182(30 \%)$ & $31(97 \%)$ & $17 \%(12 \%$ to $23 \%)$ \\
$\geq 2$ & $34(6 \%)$ & $13(41 \%)$ & $38 \%(24 \%$ to $55 \%)$ \\
Total & $614(100 \%)$ & $32(100 \%)$ & $5 \%(4 \%$ to $7 \%)$ \\
\hline
\end{tabular}

assessment, premorbid anticoagulation, a history of vomiting or confusion at stroke onset, can be used to identify those patients with minor stroke who are most likely to have ICH. One or more of these predictor variables were present in nearly all patients with ICH but were present in only $30 \%$ of all patients with minor stroke. A presentation with two or more predictor variables substantially increased the likelihood of ICH from an overall proportion of $5 \%$ to $38 \%$. Furthermore, the absence of any one of these variables made the likelihood of a recent ICH virtually negligible.

The consistency of prediction in both the derivation and validation cohorts suggests that the rule should be reliable and generalisable. Furthermore, the clinical predictors of ICH in minor stroke have face validity. Some predictors such as a history of vomiting with stroke onset, or an elevated blood pressure at assessment, have been used in previous scores, ${ }^{10} 11$ albeit to predict ICH in patients with more severe stroke. Other predictors used by these scores, such as depressed conscious state, are clearly not relevant to a cohort with minor stroke, but we have shown that milder disturbances of the sensorium such as transient confusion with stroke onset is also associated with $\mathrm{ICH}$ in minor stroke. The association between acute confusional states and ICH has also been shown in previous studies. ${ }^{18} 19$ Finally, anticoagulation use, while not a feature of previous scores, is known to be associated with a twofold to fourfold increased risk of $\mathrm{ICH}^{21}$ and anticoagulation-associated ICH now makes up a substantial proportion of all ICH in recent reports. ${ }^{22}$

This rule may be used to guide the management of patients with minor stroke, many of whom are referred to specialist outpatient clinics and consequently face some delay to undergoing investigations including brain imaging. Because there is a lack of evidence that antiplatelet agents are not harmful in acute $\mathrm{ICH}$, some referring physicians are reluctant to start these agents in patients with minor stroke until ICH has been excluded radiologically. However, the SCAN rule can be used to identify those patients in whom the likelihood of a recent ICH is virtually negligible and who should benefit from the immediate introduction of antiplatelet therapy before assessment in the specialist clinic. ${ }^{5} 6$

Conversely, the SCAN rule identifies those patients in whom the likelihood of a recent ICH may be as high as $40 \%$. If such patients present more than a week post-stroke onset and are scanned with CT, there is a risk that an $\mathrm{ICH}$ will be misdiagnosed as ischaemic stroke, ${ }^{7-9}$ resulting in the use of inappropriate or potentially harmful long-term secondary prevention with antithrombotic therapy. Although the risks of antiplatelet and warfarin use post-ICH are not absolutely defined, patients with ICH are three times more likely to have a further haemorrhagic stroke rather than an ischaemic stroke, ${ }^{23}$ and if taking aspirin or warfarin before ICH, the risk of death is increased. ${ }^{24-26}$ In particular, warfarin use post-ICH is estimated to reduce quality-adjusted life expectancy by up to 2 years. ${ }^{27}$ In view of this risk, some clinicians have advocated using MRI to screen all late presenting patients for $\mathrm{ICH}^{7}{ }^{7}$ However, this is not always practical in centres with limited access to MRI, and so the SCAN rule may be useful in prioritising the need for MRI. The SCAN rule may also be useful in prioritising the need for early CT imaging in developing countries or rural communities, where access to CT is limited. ${ }^{28}$

While several clinical scores for predicting ICH already exist, ${ }^{29-33}$ the best known of which are the Allen and Siriraj scores, ${ }^{10} 11$ these have been developed using hospitalised cohorts of patients with more disabling strokes and are not necessarily applicable to patients with minor stroke. For example, the Allen score is strongly weighted towards features of severe $\mathrm{ICH}$ including a history of loss of consciousness at stroke onset and decreased consciousness or the presence of bilateral extensor plantar responses $24 \mathrm{~h}$ post-stroke. When scores have been independently validated, they have often been criticised for being insensitive in detecting $\mathrm{ICH}^{12}{ }^{34}{ }^{35}$ possibly because cases with less debilitating stroke were missed. When the Siriraj score was applied to our cohorts with minor stroke, $29 \%$ of cases with ICH were missed in the OXVASC cohort and $87 \%$ in the hospital clinic-based cohort. In contrast to this, the SCAN rule missed no cases with ICH in OXVASC and only $7 \%$ in the hospital clinic cohort. Most importantly, the SCAN rule was validated in exactly the sort of population for which it should be most applicable-that is a cohort of late presenting outpatients.

Our study does have some limitations. First, the number of patients with ICH in the derivation cohort was small. Nevertheless, we were still able to demonstrate that several variables had a sizeable predictive effect as shown by their diagnostic ORs. While larger numbers with ICH would have made it possible to perform logistic regression analysis and further refine the model by weighting variables according to their corresponding regression coefficients, this might have produced a model that was well fitted to the derivation cohort but less generalisable to other populations with different exposures to different predictor variables. This approach would also have increased the complexity of the rule, making it less easy to remember and apply at the bedside. Second, the model works better in the derivation cohort compared with the validation cohort. This may partly be because patients were seen earlier in the derivation cohort, and so their symptom recall was probably more reliable than in the validation

Table 4 The SCAN rule

Expedite CT brain imaging or arrange MRI for late-presenting patients with minor stroke if one or more of the following are present on history taking or assessment:

(S) Severe hypertension at presentation:

systolic blood pressure $\geq 80 \mathrm{~mm} \mathrm{Hg}$ or

diastolic blood pressure $\geq 110 \mathrm{~mm} \mathrm{Hg}$

(C) Confusion

(A) Anticoagulation use before stroke

onset

(N) Nausea and vomiting 
cohort. The one patient with ICH who was missed by the SCAN rule in the validation cohort was seen 23 days after stroke onset. Blood pressure measurements in the derivation cohort were also more likely to reflect immediate post-stroke values as they were performed earlier. Elevated post-stroke systolic blood pressures appear to discriminate between ischaemic stroke and $\mathrm{ICH}^{30}{ }^{31}$ and this also holds true for patients with smaller haemorrhages and less disabling stroke as shown in the OXVASC cohort. However, blood pressure is highly variable and is more likely to be elevated just after stroke onset and fall again over the following days, ${ }^{36}$ explaining why mean systolic blood pressures were higher in the OXVASC cohort compared with the hospital clinic-based cohort. The model might have performed better in the validation cohort if the first ever blood pressure taken by primary care doctors or emergency department physicians had been available for use, rather than blood pressure recordings taken in clinic. Finally, some of the other predictor variables such as a history of vomiting and confusion might be considered as unlikely symptoms to encounter in an outpatient group. Nevertheless, these symptoms tended to be short-lived when they did occur, and there were as many inpatients as outpatients with a history of vomiting in the OXVASC cohort and as many patients in the hospital clinic-based cohort with a history of confusion as in the whole OXVASC cohort.

In conclusion, we have derived a simple rule-the SCAN rule for identifying patients with minor stroke who are most likely to have had a recent ICH. This rule is sensitive and reasonably specific on validation in an independent cohort of patients with minor stroke. Furthermore, the validation cohort was representative of those patients for whom we believe this score will be most useful, that is, patients referred for investigation and treatment in the outpatient clinic. Ideally, all patients with minor stroke should be imaged and start appropriate secondary prevention therapy on the same day as symptom onset, but until major changes take place both in the way stroke care is delivered and in the public's recognition of the need for seeking immediate medical attention for stroke symptoms, a significant number of such patients will continue to present late. The SCAN rule, while still requiring further independent validation, should be more sensitive than previous scores at identifying which patients with minor stroke are very unlikely to have had a recent ICH when starting antiplatelet therapy before outpatient brain imaging and which patients need urgent inpatient investigations or MRI to confirm a suspected ICH.

Contributors CEL collected and analysed the data and wrote the paper. JNR collected data and DB designed the hospital clinic cohort study. PMR designed the OXVASC study, and edited the paper.

Funding OXVASC is funded by the UK Medical Research Council, the National Institute of Health Research, the Stroke Association, the Dunhill Medical Trust and the Oxford Partnership Comprehensive Biomedical Research Centre, Thames Valley Primary Care Research Partnership, BUPA Foundation.

Competing interests None.

Ethical approval OXVASC and related substudies have been approved by our local ethics review committee. This study was conducted with the approval of the Oxfordshire Clinical Research Ethics Committee (CO.043).

Provenance and peer review Not commissioned; externally peer reviewed.

\section{REFERENCES}

1. Scholte op Reimer WJ, Dippel DW, Franke CL, et al. Quality of hospital and outpatient care after stroke or transient ischemic attack: insights from a stroke survey in the Netherlands. Stroke 2006;37:1844-9.

2. Roebers S, Wagner M, Ritter MA, et al. Attitudes and current practice of primary care physicians in acute stroke management. Stroke 2007;38:1298-303.

3. Department of Health. National stroke strategy. London: Department of Health 2007.
4. Intercollegiate Stroke Working Party. National Sentinel Stroke Audit. Phase 1 organizational audit. London: Royal College of Physicians, 2008.

5. Rothwell PM, Giles MF, Chandratheva A, et al. Effect of urgent treatment of transient ischaemic attack and minor stroke on early recurrent stroke (EXPRESS study): a prospective population-based sequential comparison. Lancet 2007; 370:1432-42.

6. Kennedy J, Hill MD, Ryckborst KJ, et al. Fast assessment of stroke and transient ischaemic attack to prevent early recurrence (FASTER): a randomised controlled pilo trial. Lancet Neurol 2007:6:961-9.

7. Wardlaw JM, Keir SL, Dennis MS. The impact of delays in computed tomography of the brain on the accuracy of diagnosis and subsequent management in patients with minor stroke. J Neurol Neurosurg Psychiatry 2003;74:77-81.

8. Hung TP, Lee KY. Small intracerebral haemorrhage: a study of clinical manifestations and CT findings on 31 cases. Ann Acad Med Singapore 1985;14:22-31.

9. Dennis MS, Bamford JM, Molyneux AJ, et al. Rapid resolution of signs of primary intracerebral haemorrhage in computed tomograms of the brain. Br Med J (Clin Res Ed) 1987:295:379-81.

10. Allen CM. Clinical diagnosis of the acute stroke syndrome. O J Med 1983;52:515-23.

11. Poungvarin N, Viriyavejakul A, Komontri C. Siriraj stroke score and validation study to distinguish supratentorial intracerebral haemorrhage from infarction. BMJ 1991;302:1565-7.

12. Hawkins GC, Bonita R, Broad JB, et al. Inadequacy of clinical scoring systems to differentiate stroke subtypes in population-based studies. Stroke 1995:26:1338-42.

13. Anon. Cerebrovascular diseases: prevention, treatment, and rehabilitation. Report of a WHO meeting. World Health Organ Tech Rep Ser 1971;469:1-57.

14. Goldstein LB, Bertels C, Davis JN. Interrater reliability of the NIH stroke scale. Arch Neurol 1989;46:660-2.

15. Rothwell PM, Coull AJ, Giles MF, et al. Change in stroke incidence, mortality, casefatality, severity, and risk factors in Oxfordshire, UK from 1981 to 2004 (Oxford Vascular Study). Lancet 2004;363:1925-33.

16. Mayer TE, Schulte-Altedorneburg G, Droste DW, et al. Serial CT and MRI of ischaemic cerebral infarcts: frequency and clinical impact of haemorrhagic transformation. Neuroradiology 2000:42:233-9.

17. Anon. Diagnostic and statistical manual of mental disorders [text reviewed]. 4th edn Washington (DC): American Psychiatric Association, 2000.

18. Sheng $\mathbf{A Z}$, Shen $\mathrm{Q}$, Cordato $\mathrm{D}$, et al. Delirium within three days of stroke in a cohort of elderly patients. J Am Geriatr Soc 2006;54:1192-8.

19. Caeiro L, Ferro JM, Albuquerque R, et al. Delirium in the first days of acute stroke. J Neurol 2004:251:171-8.

20. European Society of Hypertension-European Society of Cardiology Guidelines Committee. 2003 European Society of Hypertension-European Society of Cardiology guidelines for the management of arterial hypertension. J Hypertens 2003;21:1011-53.

21. Hart RG, Tonarelli SB, Pearce LA. Avoiding central nervous system bleeding during antithrombotic therapy: recent data and ideas. Stroke 2005;36:1588-93.

22. Flaherty ML, Kissela B, Woo D, et al. The increasing incidence of anticoagulantassociated intracerebral hemorrhage. Neurology 2007;68:116-21.

23. Bailey RD, Hart RG, Benavente 0, et al. Recurrent brain hemorrhage is more frequent than ischemic stroke after intracranial hemorrhage. Neurology 2001; 56: 773-7.

24. Roquer J, Rodriguez Campello A, Gomis M, et al. Previous antiplatelet therapy is an independent predictor of 30-day mortality after spontaneous supratentorial intracerebral hemorrhage. J Neurol 2005;252:412-16.

25. Saloheimo $\mathbf{P}$, Ahonen M, Juvela S, et al. Regular aspirin-use preceding the onset of primary intracerebral hemorrhage is an independent predictor for death. Stroke 2006;37:129-33.

26. Rosand J, Eckman MH, Knudsen KA, et al. The effect of warfarin and intensity of anticoagulation on outcome of intracerebral hemorrhage. Arch Intern Med 2004;164:880-4

27. Eckman $\mathbf{M H}$, Rosand J, Knudsen KA, et al. Can patients be anticoagulated after intracerebral hemorrhage? A decision analysis. Stroke 2003:34:1710-16.

28. Joubert J, Prentice LF, Moulin T, et al. Stroke in rural areas and small communities. Stroke 2008:39:1920-8.

29. Besson G, Robert C, Hommel M, et al. Is it clinically possible to distinguish nonhemorrhagic infarct from hemorrhagic stroke? Stroke 1995;26:1205-9.

30. Massaro AR, Sacco RL, Scaff M, et al. Clinical discriminators between acute brain hemorrhage and infarction: a practical score for early patient identification. Arq Neuropsiquiatr 2002:60:185-91.

31. Sturmer T, Schlindwein G, Kleiser B, et al. Clinical diagnosis of ischemic versus hemorrhagic stroke: applicability of existing scores in the emergency situation and proposal of a new score. Neuroepidemiology 2002;21:8-17.

32. Efstathiou SP, Tsioulos DI, Zacharos ID, et al. A new classification tool for clinical differentiation between haemorrhagic and ischaemic stroke. $J$ Intern Med 2002-252:121-9.

33. Woisetschlager C, Kittler $\mathrm{H}$, Oschatz $\mathrm{E}$, et al. Out-of-hospital diagnosis of cerebral infarction versus intracranial hemorrhage. Intensive Care Med 2000;26:1561-5.

34. Weir CJ, Murray GD, Adams FG, et al. Poor accuracy of stroke scoring systems for differential clinical diagnosis of intracranial haemorrhage and infarction. Lancet 1994;344:999-1002.

35. Mader TJ, Mandel A. A new clinical scoring system fails to differentiate hemorrhagic from ischemic stroke when used in the acute care setting. J Emerg Med 1998:16:9-13.

36. Britton M, Carlsson A, de Faire U. Blood pressure course in patients with acute stroke and matched controls. Stroke 1986;17:861-4. 